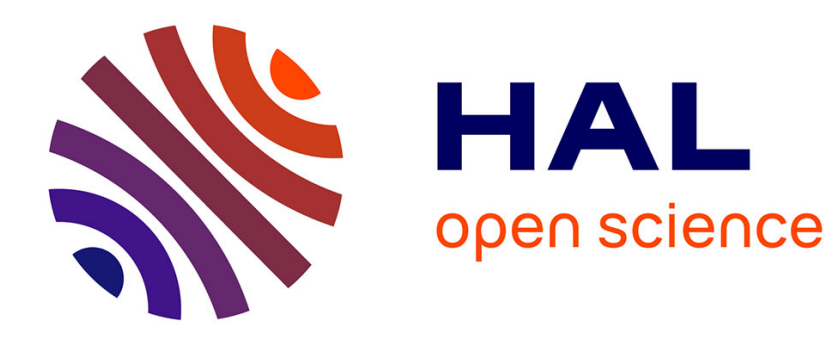

\title{
Peacocks Parametrised by a Partially Ordered Set
}

Nicolas Juillet

\section{To cite this version:}

Nicolas Juillet. Peacocks Parametrised by a Partially Ordered Set. Séminaire de Probabilités XLVIII, pp.13-32, 2016, 10.1007/978-3-319-44465-9_2 . hal-02123427

\section{HAL Id: hal-02123427 \\ https://hal.science/hal-02123427}

Submitted on 3 Apr 2020

HAL is a multi-disciplinary open access archive for the deposit and dissemination of scientific research documents, whether they are published or not. The documents may come from teaching and research institutions in France or abroad, or from public or private research centers.
L'archive ouverte pluridisciplinaire HAL, est destinée au dépôt et à la diffusion de documents scientifiques de niveau recherche, publiés ou non, émanant des établissements d'enseignement et de recherche français ou étrangers, des laboratoires publics ou privés. 


\title{
Peacocks Parametrised by a Partially Ordered Set
}

\author{
Nicolas JUILLET
}

\begin{abstract}
We indicate some counterexamples to the peacock problem for families of a) real measures indexed by a partially ordered set or b) vectorial measures indexed by a totally ordered set. This is a contribution to an open problem of the book [7] by Hirsch, Profeta, Roynette and Yor (Problem 7a-7b: "Find other versions of Kellerer's Theorem").

Case b) has been answered positively by Hirsch and Roynette in [8] but the question whether a "Markovian" Kellerer Theorem hold remains open. We provide a negative answer for a stronger version: A "Lipschitz-Markovian" Kellerer Theorem will not exist.

In case a) a partial conclusion is that no Kellerer Theorem in the sense of the original paper [14] can be obtained with the mere assumption on the convex order. Nevertheless we provide a sufficient condition for having a Markovian associate martingale. The resulting process is inspired by the quantile process obtained by using the inverse cumulative distribution function of measures $\left(\mu_{t}\right)_{t \in T}$ non-decreasing in the stochastic order.

We conclude the paper with open problems.
\end{abstract}

\section{Introduction}

The rich topic investigated by Strassen [16] in his fundamental paper of 1965 was to determine whether two probability measures $\mu$ and $v$ can be the marginals of a joint law satisfying some constraints. The most popular constraint on $\operatorname{Law}(X, Y)$ is probably $\mathbb{P}(X \leq Y)=1$. In this case if $\leq$ is the usual order on $\mathbb{R}$, a necessary and sufficient condition on $\mu$ and $v$ to be the marginals of $(X, Y)$ is $F_{\mu} \geq F_{v}$, where $F_{\eta}$

Nicolas JUILLET

Institut de Recherche Mathématique Avancée, UMR 7501, Université de Strasbourg et CNRS, 7 rue René Descartes, 67000 Strasbourg, France, e-mail: nicolas . juil let @math. unistra. fr 
denotes the cumulative distribution function of $\eta$. Actually if we note $G_{\eta}$ the quantile function of $\eta$, the random variable $\left(G_{\mu}, G_{v}\right)$ answers the question. Recall that the quantile function $G_{\eta}$ is the generalised inverse of $F_{\eta}$, that is the unique nondecreasing functions on $] 0,1\left[\right.$ that is left-continuous and satisfies $\left.\left(G_{\eta}\right)_{\#} \lambda\right|_{[0,1]}=\eta$. In the case of a general family $\left(\mu_{t}\right)_{t \in T}$, the family consisting of the quantile functions $G_{\mu_{t}}$ on (] $0,1\left[,\left.\lambda\right|_{0,1[}\right)$ is also a process. It proves that measures are in stochastic order if and only if there exists a process $\left(X_{t}\right)_{t \in T}$ with $\mathbb{P}\left(t \mapsto X_{t}\right.$ is non-decreasing $)=1$ and $\operatorname{Law}\left(X_{t}\right)=\mu_{t}$ for every $t \in T$. This result is part of the mathematical folklore on couplings. We name it quantile process or Kamae-Krengel process after the authors of [12] because in this paper a generalisation for random variables valued in a partially ordered set $E$ is proven. See also [15] where it appears.

Another type of constraint on $\operatorname{Law}(X, Y)$ that is considered in Strassen article are the martingale and submartingale constraints, $\mathbb{E}(Y \mid \sigma(X))=X$ and $\mathbb{E}(Y \mid \sigma(X)) \geq X$ respectively. Strassen proved that measures $\left(\mu_{t}\right)_{t \in \mathbb{N}}$ are the marginals of a martingale $\left(X_{t}\right)_{t \in \mathbb{N}}$ if and only if the measures $\mu_{t}$ are in the so-called convex order (see Definition 2). Kellerer extended this result to processes indexed by $\mathbb{R}$ and proved that the (sub)martingales can be assumed to be Markovian. Strangely enough, but for good reasons this famous result only concerns $\mathbb{R}$-valued processes indexed by $\mathbb{R}$ or another totally ordered set, which is essentially the same in this problem. Nevertheless, Strassen-type results have from the start been investigated with partially ordered set, both for the values of the processes or for the set of indices (see [12, 13, 5]). Hence the attempt of generalising Kellerer's theorem by replacing $\mathbb{R}$ by $\mathbb{R}^{2}$ for one of the two sets is a natural open problem that has been recorded as Problem 7 by Hirsch, Profeta, Roynette and Yor in their book devoted to peacocks [7].

In parts 1 and 2 we define the different necessary concepts, state Kellerer Theorem and exam the possible generalised statement suggested in [7, Problem 7]. About Problem $7 \mathrm{~b}$ we explain in paragraph 2.2 why Kellerer could not directly apply his techniques to the case of $\mathbb{R}^{2}$-valued martingales. Problem $7 \mathrm{a}$ is the topic of the last two parts. In part 3 we exhibit counterexamples showing with several degrees of precision that one can not obtain a Kellerer theorem on the marginals of martingales indexed by $\mathbb{R}^{2}$, even if the martingales are not assumed to be Markovian. However, in part 4 we provide a sufficient condition on $\left(\mu_{t}\right)_{t \in T}$ that is inspired by the quantile process. We conclude the paper with open problems.

\section{Definitions}

Let $(T, \leq)$ be a partially ordered set. In this note, the most important example may be $\mathbb{R}^{2}$ with the partial order: $(s, t) \leq\left(s^{\prime}, t^{\prime}\right)$ if and only if $s \leq s^{\prime}$ and $t \leq t^{\prime}$. We consider probability measures with finite first moment and we simply denote this set by $\mathscr{P}\left(\mathbb{R}^{d}\right)$.

We introduce the concepts that are necessary for our paper. Martingales indexed by a partially ordered set were introduced in the 70'. Two major contributions were [3] and [17]. The theory was known under the name "two indices". 
Definition 1 (Martingale indexed by a partially ordered set). Let $\left(X_{t}\right)_{t \in T}$ be the canonical process associated to some $\mathbb{P}$ on $\left(\mathbb{R}^{d}\right)^{T}$. For every $s \in T$ we introduce $\mathscr{F}_{s}=\sigma\left(X_{r} \mid r \leq s\right)$.

A probability measure $\mathbb{P}$ on $\left(\mathbb{R}^{d}\right)^{T}$ is a martingale if and only if for every $(s, t) \in$ $T^{2}$ satisfying $s \leq t$ it holds $\mathbb{E}\left(X_{t} \mid \mathscr{F}_{s}\right)=X_{s}$. In other words it is a martingale if and only if for every $s \leq t, n \in \mathbb{N}$ and $s_{k} \leq s$ for $k \in\{0,1, \ldots, n\}$ we have $\mathbb{E}_{\mathbb{P}}\left(X_{t}\right)$ $\left.X_{s}, X_{s_{1}}, \ldots, X_{s_{n}}\right)=X_{s}$.

The convex order that we introduce now is also known under the names second stochastic order or Choquet order.

Definition 2 (Convex order). The measures $\mu, v \in \mathscr{P}\left(\mathbb{R}^{d}\right)$ are said to be in convex order if for every convex function $\varphi: \mathbb{R}^{d} \rightarrow \mathbb{R}, \int \varphi d \mu \leq \int \varphi d \nu$. This partial order is obviously transitive and we denote it by $\mu \preceq_{C} v$.

Note that in Definition 2, $\varphi$ may not be integrable but the negative part is integrable because $\varphi$ is convex.

The next concept of peacock is more recent. To our best knowledge it appeared the first time in [10] as the acronym PCOC, that is Processus Croissant pour l'Ordre Convexe. Both the writing peacock and the problem have been popularised in the book by Hirsch, Profeta, Roynette and Yor: Peacocks and Associated Martingales, with Explicit Constructions.

Definition 3 (Peacock). The family $\left(\mu_{t}\right)_{t \in T}$ is said to be a peacock if for every $s \leq t$ we have $\mu_{s} \preceq_{C} \mu_{t}$.

Because of the conditional Jensen inequality, if $\left(X_{t}\right)_{t \in T}$ is a martingale, the family $\mu_{t}=\operatorname{Law}\left(X_{t}\right)$ of marginals is a peacock. More generally if for some peacock $\left(\mu_{t}\right)_{t \in T}$ a martingale $\left(Y_{t}\right)_{t \in T}$ satisfies for every $t, \operatorname{Law}\left(Y_{t}\right)=\mu_{t}$, the martingale is said to be associated to the peacock $\left(\mu_{t}\right)_{t \in T}$.

Definition 4 (Kantorovich distance). The Kantorovich distance between $\theta$ and $\theta^{\prime} \in \mathscr{P}\left(\mathbb{R}^{d}\right)$ is

$$
W\left(\theta, \theta^{\prime}\right)=\sup _{f}\left\|\int f \mathrm{~d} \theta-\int f \mathrm{~d} \theta^{\prime}\right\|_{\mathbb{R}^{d}}
$$

where $f$ describes the set of 1-Lipschitz functions from $\mathbb{R}^{d}$ to $\mathbb{R}$.

Definition 5 (Lipschitz kernel). A kernel $k: x \mapsto \theta_{x}$ transporting $\mu$ to $v=\mu k$ is called Lipschitz if there exist a set $A \subseteq \mathbb{R}^{d}$ satisfying $\mu(A)=1$ such that $\left.k\right|_{A}$ is Lipschitz of constant 1 from $\left(A,\|\cdot\|_{\mathbb{R}^{d}}\right)$ to $\left(\mathscr{P}\left(\mathbb{R}^{d}\right), W\right)$.

As $\left(\mathscr{P}\left(\mathbb{R}^{d}\right), W\right)$ is a complete geodesic metric space a simple extension procedure that we describe now permits us to extend $k$ to a 1-Lipschitz function on $\mathbb{R}$. First the kernel $k$ seen as a map is uniformly continuous so that one can extend it in a unique way on $\bar{A}$. The connected components of $\mathbb{R} \backslash \bar{A}$ are open intervals $] a, b[$ and the linear interpolation $t \mapsto(b-a)^{-1}((t-a) k(b)+(b-t) k(a))$ is also a geodesic 
curve for the Kantorovich distance. Therefore it gives a solution for extending $k$ and making it a 1-Lipschitzian curve on $\mathbb{R}$.

To our best knowledge, the next concept is the key of all known proofs of Kellerer Theorem. Unlike Markov martingales, converging sequences of Lipschitz-Markov martingale have Markovian limits (in fact Lipschitz-Markov). In his original proof Kellerer uses a similar concept where the Kantorovich distance is replaced by the Kantorovich distance build on $d(x, y)=\min (1,|y-x|)$.

Definition 6 (Lipschitz-Markov martingale). A process $\left(X_{t}\right)_{t \in T}$ is a LipschitzMarkov martingale if it is a Markovian martingale and the Markovian transitions are Lipschitz kernels.

For surveys with examples of Lipschitz kernels and Lipschitz-Markov martin gales, one can refer to [9] or [1].

\section{The Kellerer theorem and trying to generalise it}

\subsection{Problem $7 a$}

Theorem 1 is a reformulation of Theorem 3 by Kellerer [14] in terms of the peacock terminology.

Theorem 1 (Kellerer, 1972). Let $\left(\mu_{t}\right)_{t \in T}$ be a family of integrable probability measure on $\mathscr{P}(\mathbb{R})$ indexed by the totally ordered set $T$ (for simplicity thing of $T=[0,+\infty[)$. The following statements are equivalent

1. $\mu_{t}$ is a peacock,

2. $\mu_{t}$ is associated to a martingale process $\left(X_{t}\right)_{t \in T}$,

3. $\mu_{t}$ is associated to a Markovian martingale process $\left(X_{t}\right)_{t \in T}$,

4. $\mu_{t}$ is associated to a Lipschitz-Markovian martingale process $\left(X_{t}\right)_{t \in T}$.

Note that the implications $4 \Rightarrow 3 \Rightarrow 2 \Rightarrow 1$ are obvious. Theorem 2 that we prove in Section 3 contradicts the converse implications if $T$ is merely a partially ordered set. This is a negative answer to Problem 7a that we quote: "Let $\left(X_{t, \lambda} ; t, \lambda \geq 0\right)$ be a two-parameter peacock. Does there exist an associated two-parameter martingale $\left(M_{t, \lambda} ; t, \lambda \geq 0\right)$ ?". Note that with our definition of peacock, one should read $\operatorname{Law}\left(X_{t, \lambda}\right)$ in place of $X_{t, \lambda}$.

Theorem 2. Let $(T, \leq)$ be $\{0,1\}^{2}, \mathbb{R}_{+}^{2}$ or $\mathbb{R}^{2}$ with the partial order. For every choice of $T$, we have the following:

- There exists a peacock indexed by $T$ that is not associated to a martingale,

- there exists a peacock indexed by $T$ that is associated to a martingale process but not to a Markovian martingale process,

- there exists a peacock indexed by $T$ that is associated to a Markovian martingale process but not to a Lipschitz-Markovian martingale process. 


\subsection{Problem $7 b$}

For completeness we explain what is known on Problem 7b: "Is a $\mathbb{R}^{n}$-valued peacock a $\mathbb{R}^{n}$-valued 1-martingale?", which with our notations means nothing but: Can any peacock on $\mathbb{R}^{d}$ be associated to an $\mathbb{R}^{d}$-valued martingale? Hirsch and Roynette provided a positive answer in [8].

Theorem 3. Let $\left(\mu_{t}\right)_{t \in T}$ be a family of integrable probability measures on $\mathscr{P}\left(\mathbb{R}^{d}\right)$ indexed by the totally ordered set $T$. The following statements are equivalent

1. $\mu_{t}$ is a peacock,

2. $\mu_{t}$ is associated to a martingale process $\left(X_{t}\right)_{t \in E}$.

Nevertheless it is to our knowledge still an open problem whether the full Kellerer theorem may hold in the vectorial case: Can every peacock be associated to a Markovian martingale? (equivalence of (1) and (3) in Theorem 1). We prove in Proposition 1 that (1) and (4) are not equivalent. Actually, the existence of a Lipschitz kernel for $\mu \preceq_{C} v$ is an essential step of each known proof of Kellerer Theorem, but for dimension $d>1$ it does not exist for any pairs. This fact was very likely known by Kellerer (see the last paragraph of the introduction of $[14]^{1}$ ). We provide a short proof of it.

Proposition 1. There exists a peacock $\left(\mu_{t}\right)_{t \in T}$ indexed by $T=\{0,1\}$ and with $\mu_{t} \in$ $\mathscr{P}\left(\mathbb{R}^{2}\right)$ that is not associated to any Lipschitz-Markov martingale.

As a trivial corollary, the same also holds for $T=\left[0,+\infty\left[\right.\right.$ defining $\mu_{t}=\mu_{0}$ on $\left[0,1\left[\right.\right.$ and $\mu_{t}=\mu_{1}$ for $t \in[1,+\infty[$.

Proof. Let $\mu_{0}=\left.\lambda\right|_{[0,1]} \times \delta_{0} \in \mathscr{P}\left(\mathbb{R}^{2}\right)$ and $k$ the dilation $(x, 0) \mapsto \frac{1}{2}\left(\delta_{(x, f(x))}+\right.$ $\left.\delta_{(x,-f(x))}\right)$. Let $\mu_{1}$ be $\mu_{0} k$. If $\mu_{1}=\mu_{0} k^{\prime}$ for another dilation $k^{\prime}$, the projection of $k^{\prime}$ on the $O x$-axis must be identity so that $k^{\prime}=k$. We choose a non continuous function $f$ as for instance $f=\chi_{[1 / 2,1]}$, and the proof is complete because $k$ is not a Lipschitz kernel.

\section{Proof of Theorem 2}

In the three examples, we define a peacock on $T=\left\{0,1,1^{\prime}, 2\right\} \equiv\{0,1\}^{2}$ where the indices $1,1^{\prime}$ stand for the intermediate elements, $0 \equiv(0,0)$ is the minimal and $2 \equiv$ $(1,1)$ the maximal element. One will easily check that $\left(\mu_{i}\right)_{i \in T}$ is really a peacock from the fact that we indicate during the proof martingale transitions between $\mu_{0}$ and $\mu_{1}, \mu_{1^{\prime}}$ as well as between $\mu_{1}, \mu_{1^{\prime}}$ and $\mu_{2}$.

To complete the statement of Theorem 2 we need to explain what are the peacocks for $T=\mathbb{R}_{+}^{2}$ or $T=\mathbb{R}^{2}$. In fact for $(s, t) \in\{0,1\}^{2}$, the measures $\mu_{s, t}$ are defined

\footnotetext{
${ }^{1}$ Kellerer: "[... ], während die Übertragung der im zweiten Teil enthaltenen Ergebnisse etwa auf den mehrdimensionalen Fall ein offenes Problem darstellt"
} 
exactly as in the three following constructions, and the peacock is extended in the following way

$$
\mu_{s, t}= \begin{cases}\mu_{1,1} & \text { if }(s, t) \geq(1,1) \\ \mu_{1,0} & \text { if } s \geq 1 \text { and } t<1 \\ \mu_{0,1} & \text { if } t \geq 1 \text { and } s<1 \\ \mu_{0,0} & \text { otherwise: } \max (s, t)<1\end{cases}
$$

With this bijection it is a direct check that the results for $\left\{0,1,1^{\prime}, 2\right\}$ will be transposed to the other sets of indices. Note that if a martingale $\left(X_{s, t}\right)$ is defined for $(s, t) \in\{0,1\}^{2}$ it is extended in the same way as the peacock. For instance $X_{s, t}=X_{1,1}$ if $(s, t) \geq(1,1)$.

The three constructions are illustrated by figures where the amount of transported mass from $x$ to $y$ is the label of the arrow from $x$ at time $i$ to $y$ at time $j$ where $i \leq j$ (and $(i, j)$ is not $(0,2)$ ). In order to write an integer we prefer to label with a multiple of the mass (factor 6 in Figure 3.1 and 3.2, and 12 in Figure 3.3).

\subsection{A peacock not associated to a martingale}

We introduce the following peacock $\left(\mu_{t}\right)_{t \in T}$ :

$$
\left\{\begin{aligned}
6 \mu_{0} & =3 \delta_{-1}+3 \delta_{1} \\
6 \mu_{1} & =2 \delta_{-2}+4 \delta_{1} \\
6 \mu_{2} & =2 \delta_{0}+2 \delta_{-2}+2 \delta_{2} \\
6 \mu_{1^{\prime}} & =4 \delta_{-1}+2 \delta_{2}
\end{aligned}\right.
$$

Fig. 1 The martingale associated to $\left(\mu_{t}\right)_{t \in\{0,1,2\}}$ in paragraph 3.1.

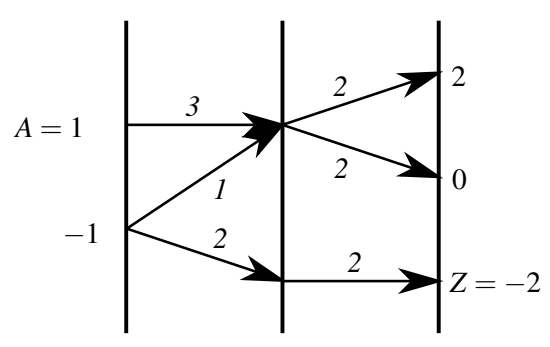

Note that the measures $\mu_{0}$ and $\mu_{2}$ are symmetric and $\mu_{1}$ and $\mu_{1^{\prime}}$ are obtained from the other by symmetry. On Figure 3.1 we represent the (sub)peacock $\left(\mu_{t}\right)_{t \in\{0,1,2\}}$. It is easily seen that every martingale transition is uniquely determined. There exists an associated martingale that is forced to have the law 


$$
(1 / 3) \delta_{-1,-2,-2}+1 / 12\left(\delta_{-1,1,0}+\delta_{-1,1,2}\right)+1 / 4\left(\delta_{1,1,0}+\delta_{1,1,2}\right) .
$$

Hence, the law of the coupling between $\mu_{0}$ and $\mu_{2}$ is

$$
\pi=(1 / 3) \delta_{-1-2}+1 / 12\left(\delta_{-1,0}+\delta_{-1,2}\right)+1 / 4\left(\delta_{1,0}+\delta_{1,2}\right)
$$

Observe that the coefficient of $\delta_{1,-2}$ is zero. In other words, no mass is transported from 1 at time 0 (point $A$ on Figure 3.1) to -2 at time 2 (point $Z$ ). For the peacock $\left(\mu_{t}\right)_{t \in\left\{0,1^{\prime}, 2\right\}}$ the coupling between $\mu_{0}$ and $\mu_{2}$ is obtained by symmetry from $\pi$. Thus some mass is transported from $A$ to $Z$. Hence, there does not exist a martingale associated to both (sub)peacocks. Therefore, one can not associate a martingale to $\left(\mu_{t}\right)_{t \in T}$.

\subsection{A martingale not associated to a Markovian martingale}

For the second item of Theorem 2 we introduce a slight modification of the previous peacock where $\mu_{0}$ does not change but the final peacock is concentrated on $\{-5,0,5\}$ instead of $\{-2,0,2\}$.

$$
\left\{\begin{aligned}
6 \mu_{0} & =3 \delta_{-1}+3 \delta_{1} \\
6 \mu_{1} & =\delta_{-5}+5 \delta_{1} \\
6 \mu_{2} & =2 \delta_{0}+2 \delta_{-5}+2 \delta_{5} \\
6 \mu_{1^{\prime}} & =5 \delta_{-1}+\delta_{5}
\end{aligned}\right.
$$

As in paragraph 3.1 the peacocks $\left(\mu_{t}\right)_{t \in\{0,1,2\}}$ and $\left(\mu_{t}\right)_{t \in\left\{0,1^{\prime}, 2\right\}}$ are symmetric and the proof is similar. But the two symmetric martingales associated to the peacocks indexed by $\{0,1,2\}$ and $\left\{0,1^{\prime}, 2\right\}$ are now unique only because one asks them to be Markovian. Let us see what is the first one that we call $\left(X_{t}\right)_{t \in\{0,1,2\}}$. It is obtained as the Markov composition of $\operatorname{Law}\left(X_{0}, X_{1}\right)$ and $\operatorname{Law}\left(X_{1}, X_{2}\right)$ that are uniquely determined as follows:

$$
\operatorname{Law}\left(X_{0}, X_{1}\right)=(1 / 2) \delta_{1,1}+(1 / 6)\left(\delta_{-1,-5}+2 \delta_{-1,1}\right)
$$

and

$$
\operatorname{Law}\left(X_{1}, X_{2}\right)=(1 / 6) \delta_{-5,-5}+(1 / 6)\left(\delta_{1,-5}+2 \delta_{1,0}+2 \delta_{1,5}\right) .
$$

This can be read on Figure 3.2 where for the law of the Markovian martingale it remains to explain that at time 1 the mass is distributed independently from the past. For instance the coefficient of $\delta_{-1,1,5}$ is computed in the following way

$$
\mathbb{P}\left(X_{0}=-1\right) \mathbb{P}_{X_{0}=-1}\left(X_{1}=1\right) \mathbb{P}_{X_{1}=1}\left(X_{2}=5\right)=\frac{1}{2} \frac{2}{3} \frac{2}{5}=\frac{2}{15} \text {. }
$$

Finally, 
Fig. 2 The transition kernels of $\left(X_{t}\right)_{t \in\{0,1,2\}}$ in paragraph 3.2 .

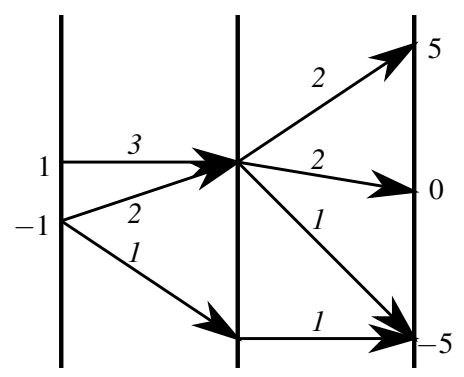

$$
\begin{aligned}
\operatorname{Law}\left(X_{0}, X_{1}, X_{2}\right)= & (1 / 6) \delta_{-1,-5,-5}+(1 / 15) \delta_{-1,1,-5}+(2 / 15) \delta_{-1,1,0} \\
& +(2 / 15) \delta_{-1,1,5}+(1 / 10) \delta_{1,1,-5}+(1 / 5) \delta_{1,1,0}+(1 / 5) \delta_{1,1,5}
\end{aligned}
$$

Observe that in $\operatorname{Law}\left(X_{0}, X_{2}\right)$ the coefficient of $\delta_{1,-5}$ and $\delta_{-1,5}$ are $1 / 10$ and $2 / 15$ respectively. Hence the measure is not symmetric, which completes the first part of the proof.

For the second part of the proof, it is enough to twist the composition of $\operatorname{Law}\left(X_{0}, X_{1}\right)$ and $\operatorname{Law}\left(X_{1}, X_{2}\right)$ at time 1 in a way that $\operatorname{Law}\left(X_{0}, X_{2}\right)$ becomes symmetric. This occurs exactly if

$$
\mathbb{P}\left(\left(X_{0}, X_{2}\right)=(1,0)\right)=\mathbb{P}\left(\left(X_{0}, X_{2}\right)=(-1,0)\right)=1 / 6
$$

because the space of martingales associated to $\mu_{0}, \mu_{2}$ depends only on one real parameter. The whole martingale $\left(X_{t}\right)_{t \in\{0,1,2\}}$ can be parametrised by the conditional law $\operatorname{Law}_{\left(X_{0}, X_{1}\right)=(1,1)}\left(X_{2}\right)$. We set $\operatorname{Law}_{\left(X_{0}, X_{1}\right)=(1,1)}\left(X_{2}\right)=\alpha^{\theta}$ defined as $\theta \alpha^{1}+(1-$ $\theta) \alpha^{0}$, where $\alpha^{0}=(3 / 5) \delta_{5}+(2 / 5) \delta_{-5}$ and $\alpha^{1}=(1 / 5) \delta_{5}+(4 / 5) \delta_{0}$ are the extreme admissible points. Notice that the Markovian composition would corresponds to the choice $\theta=1 / 2$ because $\operatorname{Law}_{X_{1}=1}\left(X_{2}\right)=\alpha^{1 / 2}$. We have

$$
\begin{aligned}
\mathbb{P}\left(\left(X_{0}, X_{2}\right)=(1,0)\right) & =\mathbb{P}\left(\left(X_{0}, X_{1}, X_{2}\right)=(1,1,0)\right) \\
& =\frac{1}{2} \mathbb{P}\left(X_{0}, X_{1}\right)=(1,1)\left(X_{2}=0\right) \\
& =\frac{1}{2}\left(\frac{4}{5} \theta\right)
\end{aligned}
$$

Thus we choose $\theta=5 / 12$, which permits us to complete the proof.

\subsection{A Markovian martingale not associated to a Lipschitz-Markov martingale}

For the last item of Theorem 2 the peacocks $\left(\mu_{t}\right)_{t \in\{0,1,2\}}$ and $\left(\mu_{t}\right)_{t \in\left\{0,{ }^{\prime} 1,2\right\}}$ are not symmetric in any way. That is why we represent both peacocks in Figure 3.3. 


$$
\left\{\begin{aligned}
12 \mu_{0} & =6 \delta_{1}+6 \delta_{2} \\
12 \mu_{1} & =2 \delta_{0}+6 \delta_{1}+4 \delta_{3} \\
12 \mu_{2} & =5 \delta_{0}+6 \delta_{2}+\delta_{6} \\
12 \mu_{1^{\prime}} & =10 \delta_{1}+\delta_{2}+\delta_{6}
\end{aligned}\right.
$$

Let see that there is a Markovian martingale associated to this peacock. Let us define the following joint laws:

$$
\left\{\begin{aligned}
\operatorname{Law}\left(X_{0}, X_{1}\right) & =(12)^{-1}\left(6 \delta_{1,1}+2 \delta_{2,0}+4 \delta_{2,3}\right) \\
\operatorname{Law}\left(X_{0}, X_{1^{\prime}}\right) & =(12)^{-1}\left(6 \delta_{1,1}+4 \delta_{2,1}+\delta_{2,2}+\delta_{2,6}\right) \\
\operatorname{Law}\left(X_{1}, X_{2}\right) & =(12)^{-1}\left(2 \delta_{0,0}+3 \delta_{1,0}+3 \delta_{1,2}+3 \delta_{3,2}+\delta_{3,6}\right) \\
\operatorname{Law}\left(X_{1^{\prime}}, X_{2}\right) & =(12)^{-1}\left(5 \delta_{1,0}+5 \delta_{1,2}+\delta_{2,2}+\delta_{6,6}\right) .
\end{aligned}\right.
$$

Fig. 3 The peacocks

$\left(\mu_{t}\right)_{t \in\{0,1,2\}}$ and $\left(\mu_{t}\right)_{t \in\left\{0,,^{\prime}, 2\right\}}$.
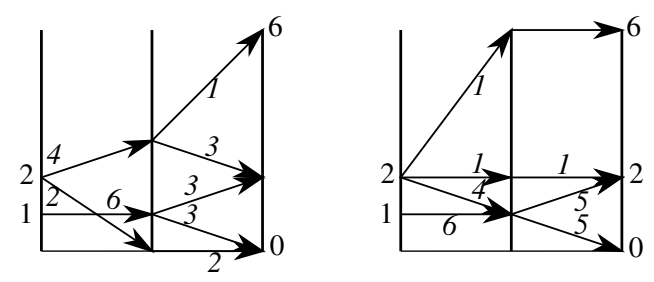

Assuming that the composition at times 1 and $1^{\prime}$ are Markovian, we obtain the same joint law $\operatorname{Law}\left(X_{0}, X_{2}\right)$. For this, as in paragraph 3.2 it suffices to compute one parameter of it in two manners. Let us do it for $\mathbb{P}\left(\left(X_{0}, X_{2}\right)=(2,2)\right)$ :

$$
\left\{\begin{array}{l}
\mathbb{P}\left(\left(X_{0}, X_{2}\right)=(2,2)\right)=\mathbb{P}\left(\left(X_{0}, X_{1}, X_{2}\right)=(2,3,2)\right)=\frac{1}{2} \frac{2}{3} \frac{3}{4} \\
\mathbb{P}\left(\left(X_{0}, X_{2}\right)=(2,2)\right)=\mathbb{P}\left(\left(X_{0}, X_{1^{\prime}}, X_{2}\right)=(2,2,2)\right)=\frac{1}{2} \frac{1}{6} 1+\frac{1}{2} \frac{2}{3} \frac{1}{2} .
\end{array}\right.
$$

Finally, we have proved that $X_{1}$ and $X_{1^{\prime}}$ can be defined on the same probability space together with $X_{0}$ and $X_{2}$.

Note that the previous martingale is not Lipschitz-Markov because the Kantorovich distance between $\operatorname{Law}_{X_{0}=1}\left(X_{1}\right)=\delta_{1}$ and $\operatorname{Law}_{X_{0}=2}\left(X_{1}\right)=1 / 3 \delta_{0}+2 / 3 \delta_{2}$ is $5 / 3$, which is strictly greater that $|1-0|=1$. In (2), the marginal and martingale constraints uniquely determine all of the law apart from $\operatorname{Law}\left(X_{0}, X_{1}\right)$. It can be parametrised by

$$
\operatorname{Law}\left(X_{0}, X_{1}\right)=\theta \pi^{1}+(1-\theta) \pi^{0}
$$

where $\pi^{1}=(12)^{-1}\left(6 \delta_{1,1}+2 \delta_{2,0}+4 \delta_{2,3}\right)$ corresponds to the joint law in (2) and

$$
\pi^{0}=(12)^{-1}\left(2 \delta_{1,0}+3 \delta_{1,1}+1 \delta_{1,3}+0 \delta_{2,0}+3 \delta_{2,1}+3 \delta_{2,3}\right) .
$$


The kernel is Lipschitz if and only if $\theta \in[0,1 / 2]$. However we will not need to prove it because if $\theta \neq 1$, some mass is transported from 1 to 3 and part of this mass finishes in 6 at time $t=2$. This leads to a joint law $\operatorname{Law}\left(X_{0}, X_{2}\right)$ that can not be associated to the peacock $\left(\mu_{t}\right)_{t \in\{0,1,2\}}$ on the right part of Figure 3.3. For the unique martingale law associated to this peacock, no mass is transported from 1 at time $t=0$ to 6 at time $t=2$.

\section{A positive result}

The aim of this section is to furnish sufficient conditions for Problem 7a. Under the hypothesis of Theorem 4, any peacock is associated to a martingale. Under the hypothesis of Theorem 5, this martingale is Markovian. Other examples are given by Hirsch, Profeta, Roynette and Yor in Exercise 2.3 of [7].

\subsection{Disintegration of a measure in $\{\mu \in \mathscr{P}: \mathbb{E}(\mu)=0\}$.}

As in Choquet theorem, even if $\{\mu \in \mathscr{P}: \mathbb{E}(\mu)=0\}$ is a noncompact set, any element can be decomposed as a mean of the extreme points. According to Douglas theorem [4] the extreme points are exactly the positive measures $\mu$ such that the affine functions are dense in $L^{1}(\mu)$. Hence, the extreme points are the diatomic measures $\theta_{a, b}$ with $a \leq 0 \leq b$ and $\theta_{a, b}=\frac{b}{b-a} \delta_{a}+\frac{-a}{b-a} \delta_{b}$. The decomposition is not unique as illustrated by $1 / 6\left(4 \theta_{-1,1}+2 \theta_{-2,2}\right)=1 / 6\left(3 \theta_{-1,2}+3 \theta_{-2,1}\right)$.

However one can give a canonical decomposition of $\mu$. It relies on the order of its quantiles. It seems classical but we could not cite it from the literature. Hence we present some intuitive facts as consequences of the theory developed in [2] about the minimal shadow of positive measures into other measures. For every $q \in[0,1]$, the set

$$
F(\mu, q)=\left\{\eta \mid \eta \leq \mu \text { and } \eta(\mathbb{R})=q \text { and } \int x \mathrm{~d} \eta(x)=0\right\}
$$

has a minimal element for the convex order. We call it $S^{\mu}(q)$. It is the shadow of $q \delta_{0}$ in $\mu$ as defined in Lemma 4.6 of [2]. This measure can be described as in Example 4.7 of [2]: It is the unique measure $\eta \leq \mu$ of mass $q$ and expectation 0 that can be written

$$
\eta=\left.\mu\right|_{] f(q), g(q)[}+a \delta_{f(q)}+b \delta_{g(q)} .
$$

In other words it is the restriction of $\mu$ on a quantile interval

$$
S^{\mu}(q)=\left.\left(G_{\mu}\right) \# \lambda\right|_{\left[q_{0}, q_{1}\right]}
$$


where we recall that $G_{\mu}$ is the quantile function defined before Section 1. In particular $q_{1}-q_{0}=q$.

Let $f^{\mu}$ and $g^{\mu}$ denote the functions

$$
f^{\mu}: q \mapsto \max \left(\operatorname{spt}\left(S^{\mu}(q)\right)\right) \text { and } g^{\mu}: q \mapsto \min \left(\operatorname{spt}\left(S^{\mu}(q)\right)\right) .
$$

We have the following properties

- If $q \leq q^{\prime}$ it holds $S^{\mu}(q) \leq S^{\mu}\left(q^{\prime}\right)$,

- The function $g^{\mu}$ is left-continuous and nondecreasing,

- The function $f^{\mu}$ is left-continuous and nonincreasing.

Note that $\left[g^{\mu}(q), f^{\mu}(q)\right]$ is the smallest closed interval $[f(q), g(q)]$ of full mass for $S^{\mu}(q)$ and it is the unique choice if one demands that $q \mapsto g(q)-f(q)$ is leftcontinuous in (3). We will call it the interval of quantile $q$ or the $q$-interval.

Let us now introduce a measure $\pi$ on $\mathbb{R}^{2}$ such that for every $q \in[0,1]$ the first

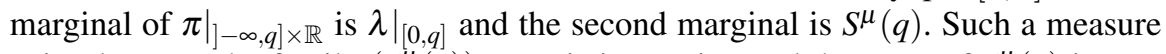
exists because the family $\left(S^{\mu}(q)\right)_{q \in[0,1]}$ is increasing and the mass of $S^{\mu}(q)$ is $q$. It is easy to check that $\left(\theta_{f^{\mu}(q), g^{\mu}(q)}\right)_{q \in[0,1]}$ is an admissible disintegration of $\pi$ with respect to $\left.\lambda\right|_{[0,1]}$ and it is the only disintegration $\left(\theta_{q}\right)_{q \in[0,1]}$ such that $q \mapsto \theta_{q}$ is left-continuous for the weak topology. Finally we have obtained a canonical representation of $\mu$. It writes

$$
\mu=\int_{0}^{1} \theta_{f^{\mu}(q), g^{\mu}(q)} \mathrm{d} q
$$

where $-f^{\mu}$ and $g^{\mu}$ are the unique nondecreasing and left-continuous functions with $S^{\mu}(q)\left(\left[f^{\mu}(q), g^{\mu}(q)\right]\right)=q$. Note that as usual for a Choquet decomposition, the equation (4) has to be understood in the weak sense. For instance

$$
\mu(A)=\int_{0}^{1} \theta_{f^{\mu}(q), g^{\mu}(q)}(A) \mathrm{d} q
$$

for every measurable $A$.

Remark 1. In this article we will sometime simply write $F_{t}, G_{t}$ and $\left[f^{t}(q), g^{t}(q)\right]$ in place of $F_{\mu_{t}}, G_{\mu_{t}}$ and $\left[f^{\mu_{t}}(q), g^{\mu_{t}}(q)\right]$ respectively.

\subsubsection{Diatomic convex order}

Let $\mu$ and $v$ be probability measures on $\mathbb{R}$ with expectation zero. We introduce the order $\preceq_{D C}$ with $\mu \preceq_{D C} v$ if and only if

$$
\forall q \in[0,1],\left[f^{\mu}(q), g^{\mu}(q)\right] \subseteq\left[f^{v}(q), g^{v}(q)\right] .
$$

and call it the diatomic convex order. There exists a unique martingale law $\pi_{q}$ be-

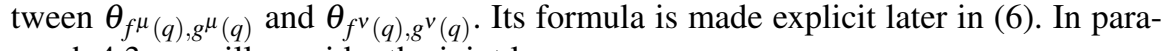
graph 4.3 we will consider the joint law 


$$
\pi=\int_{0}^{1} \pi_{q} \mathrm{~d} q
$$

with marginals $\mu$ and $v$. It is a martingale so that $\mu \preceq_{D C} v$ implies $\mu \preceq_{C} v$.

In the special case of symmetric measure, the order can be defined similarly as the stochastic order using the positive cone of even functions that are non-decreasing on $\mathbb{R}_{+}$in place of the cone of convex functions. However, I could not find an appropriate cone for defining $\preceq_{D C}$ in the general case.

\subsection{Peacocks consisting of extreme elements}

In this subsection we consider Problem 7a for peacocks $\left(\mu_{t}\right)_{t \in T}$ where every $\mu_{t}$ is an extreme element $\theta_{a, b}$. Observe that in this case, $\theta_{a, b} \preceq_{C} \theta_{a^{\prime}, b^{\prime}}$ is equivalent to $[a, b] \subseteq\left[a^{\prime}, b^{\prime}\right]$, and as these two intervals are the $q$-intervals for every $q$, the relation $\theta_{a, b} \preceq_{C} \theta_{a^{\prime}, b^{\prime}}$ is equivalent to $\theta_{a, b} \preceq_{D C} \theta_{a^{\prime}, b^{\prime}}$. The set $\Pi_{M}\left(\theta_{a, b}, \theta_{a^{\prime}, b^{\prime}}\right)$ of martingales associated to a peacock of cardinal two is restricted to one element:

$$
\begin{cases}\delta_{0} \times \theta_{a^{\prime}, b^{\prime}} & \text { if } a=b \\ \frac{b}{b-a}\left(\frac{b^{\prime}-a}{b^{\prime}-a^{\prime}} \delta_{a, a^{\prime}}+\frac{a-a^{\prime}}{b^{\prime}-a^{\prime}} \delta_{a, b^{\prime}}\right)+\frac{-a}{b-a}\left(\frac{b^{\prime}-b}{b^{\prime}-a^{\prime}} \delta_{b, a^{\prime}}+\frac{b-a^{\prime}}{b^{\prime}-a^{\prime}} \delta_{b, b^{\prime}}\right) & \text { otherwise. }\end{cases}
$$

We consider the totally ordered case before the general case.

\subsubsection{Totally ordered $(T, \leq)$}

For $s \leq t$, the two-marginals joint law $\pi_{s t}$ between $\mu_{s}$ and $\mu_{t}$ is unique and has formula (6). Hence, for $s \leq t \leq u$, the Markovian and in fact any composition of $\pi_{s t}$ and $\pi_{t u}$ is a martingale law with marginals $\mu_{s}$ and $\mu_{u}$. Thus, it is $\pi_{s u}$ and the two-marginals joint laws $\left(\pi_{s t}\right)_{(s \leq t)}$ constitute a coherent family for the Markovian composition. Thus, there exists a Markovian martingale $\left(X_{t}\right)_{t \in T}$ with the wanted marginals and its law is the unique one among the associated martingales.

\subsubsection{Partially ordered $(T, \leq)$}

It is less direct to associate a martingale when $T$ is not totally ordered. It is no longer enough to check that the two-marginals laws constitute a coherent family. All finite families of marginals would have to be considered, also with elements noncomparable for $\preceq_{C}$ and their joint law can not uniquely be determined by the constraints of the problem.

Let us first reduce the problem to $\left(\mathbb{R}_{+}^{2}, \leq\right)$. We can map $t \in T$ to the element $\Phi(t)=(a, b) \in \mathbb{R}_{+}^{2}$ defined by $\mu_{t}=\theta_{-a, b}$. If we associate a Markovian martingale 
$\left(M_{x, y}\right)_{(x, y) \in \mathbb{R}_{+}^{2}}$ to $\left(\mu_{x, y}\right)_{(x, y) \in \mathbb{R}_{+}^{2}}$ with $\mu_{x, y}=\theta_{-x, y}$, it is easy to check that $\left(M_{\Phi(t)}\right)_{t \in T}$ is a Markovian martingale associated to $\left(\mu_{t}\right)_{t \in T}$.

A martingale associated to $\left(\mu_{x, y}\right)_{(x, y) \in \mathbb{R}_{+}^{2}}$ is the following: Consider the Wiener measure on $\mathscr{C}\left(\mathbb{R}_{+}\right)$and let $M_{x, y}$ be the random variable

$$
M_{x, y}=y \cdot \mathbb{1}_{\left\{\tau_{y}<\tau_{-x}\right\}}-x \cdot \mathbb{1}_{\left\{\tau_{-x}<\tau_{y}\right\}},
$$

where $\tau_{z}$ is the hitting time of $z \in \mathbb{R}$. It is easy to check that $\left(M_{x, y}\right)_{(x, y) \in \mathbb{R}_{+}^{2}}$ is a Markovian martingale associated to $\left(\mu_{x, y}\right)_{(x, y) \in \mathbb{R}_{+}^{2}}$. For every restriction of the peacock to indices in a totally ordered set, the restriction of this martingale have the law described in paragraph 4.2.1.

Remark 2. The referee of this paper suggested to look at a peacock constructed from a reference measure $\zeta$ of barycenter 0 and defined by $\omega_{-a, b}=\left(\left.\zeta\right|_{]-\infty,-a] \cup[b,+\infty[}\right)+$ $\xi_{a, b}$ where $\xi$ is the measure concentrated on $\{a, b\}$ with the same mass and barycenter as $\left.\zeta\right|_{|a, b|}$. Note that in the case $\zeta=\delta_{0}$ it holds $\omega_{-a, b}=\theta_{-a, b}$.

The construction of this section generalises as follows. Let $B_{t}$ be a Brownian motion with $\operatorname{Law}\left(B_{0}\right)=\zeta$ and for every $(a, b) \in \mathbb{R}_{+}^{2}$ let $\tau_{a, b}$ be the hitting time of ]$-\infty,-a] \cup\left[b,+\infty\left[\right.\right.$. As $\operatorname{Law}\left(B_{\tau_{a, b}}\right)=\omega_{-a, b}$, we can simply associate the martingale $\left(B_{\tau_{a, b}}\right)_{(a, b) \in \mathbb{R}_{+}^{2}}$ to the peacock $\left(\omega_{-a, b}\right)_{(a, b) \in \mathbb{R}_{+}^{2}}$.

Notice finally that the measures $\left(\omega_{-a, b}\right)_{(a, b) \in \mathbb{R}_{+}^{2}}$ are not non decreasing for $\preceq_{D C}$, as can be easily seen if $\zeta$ is uniform on $[-1,1]$. Hence Theorem 4 does not apply.

\subsection{A positive result for peacocks indexed by a partially ordered set}

In paragraph 4.3.2, for families of measures in the diatomic convex order we introduce the process similar to the quantile process in the martingale setting. We call it the quantile martingale. Recall that the quantile process relies on the quantile coupling, that is actually the model of (5) in the nonmartingale setting. In place of measures $\theta_{a, b}$ the extreme elements are Dirac masses that are parametrised by a quantile $q \in] 0,1$ [ for the two marginals. The quantile coupling couples them using the same parameter $q$ in both disintegrations, as (5) does in the martingale case.

Theorem 5 states under which condition the quantile coupling has the Markov property. For completeness we start in Subsection 4.3.1 with the same question for the quantile process.

\subsubsection{Characterisation of Markovian Kamae-Krengel processes}

We state the result on the quantile process and its relation to the stochastic order explained in the introduction of the present paper.

Proposition 2. Let $\left(\mu_{t}\right)_{t \in T}$ be a family of real probability measures indexed by a partially order set $(T, \leq)$. The following statements are equivalent. 
- The map $t \mapsto \mu_{t}$ is nondecreasing in stochastic order,

- the associated quantile process $t \mapsto X_{t}$ is almost surely nondecreasing.

The following proposition characterises the Markovian quantile processes.

Proposition 3. The quantile process is Markovian if and only if the following criterion is satisfied: for every $s \leq t \leq u$ and $\left.q<q^{\prime} \in\right] 0,1$, the conjunction of conditions

$$
\left\{\begin{array}{l}
G_{\mu_{s}}(q)<G_{\mu_{s}}\left(q^{\prime}\right) \\
G_{\mu_{t}}(q)=G_{\mu_{t}}\left(q^{\prime}\right)
\end{array}\right.
$$

implies $G_{\mu_{u}}(q)=G_{\mu_{u}}\left(q^{\prime}\right)$. In other words $G_{\mu_{t}}(q)=G_{\mu_{t}}\left(q^{\prime}\right)$ implies $\left\{G_{\mu_{s}}(q)=\right.$ $G_{\mu_{s}}\left(q^{\prime}\right)$ or $\left.G_{\mu_{u}}(q)=G_{\mu_{u}}\left(q^{\prime}\right)\right\}$.

Proof. Assume that the property on the quantile function holds. Recall that $\mathscr{F}_{t}$ is the $\sigma$-algebra generated by all $X_{s}$ where $s \leq t$. The Markov property holds if $\mathbb{E}\left(f\left(X_{u}\right) \mid \mathscr{F}_{t}\right)=\mathbb{E}\left(f\left(X_{u}\right) \mid \sigma\left(X_{t}\right)\right)$ for every bounded measurable function $f$ and $t \leq u$ elements of $T$. Let now $t$ and $u$ be fixed. It is enough to prove that for any $k \in \mathbb{N}$ and $s_{1}, \ldots, s_{k} \leq t$ the random vectors $\left(X_{s_{1}}, \ldots, X_{s_{k}}\right)$ and $X_{u}$ are conditionally independent given $X_{t}$. For a family of conditional probabilities $\left(\mathbb{P}_{X_{t}=y}\right)_{y \in \mathbb{R}}$ it is sufficient to prove that given real numbers $\left(x_{1}, \ldots, x_{k}\right)$ and $z$ the events

$$
A=\left\{X_{s_{1}} \leq x_{1}, \ldots, X_{s_{k}} \leq x_{k}\right\} \text { and } B=\left\{X_{u} \leq z\right\}
$$

are independent under $\mathbb{P}_{X_{t}=y}$ for all $y$. We will define such a family. Recall that $\mathbb{P}$ is defined as $\int_{0}^{1} \mathbb{P}_{q} \mathrm{~d} q$ where the law of $\left(X_{s_{1}}, \ldots, X_{s_{k}}, X_{t}, X_{u}\right)$ under $\mathbb{P}_{q}$ is simply the Dirac mass in $\left(G_{s_{1}}(q), \ldots, G_{s_{k}}(q), G_{t}(q), G_{u}(q)\right)$.

The events $\left\{X_{t}=y\right\}$ is of type $\{q \in] 0,1\left[, G_{t}(q)=y\right\}$, that is $\left.] q^{-}, q^{+}\right]$or $] q^{-}, 1[$ where $q^{+}=F_{t}(y)$ and $q^{-}=\lim _{\varepsilon \rightarrow 0^{+}} F_{t}(y-\varepsilon)$. Recall that $\mu(y)=q^{+}-q^{-}$. Thus $\left(\mathbb{P}_{X_{t}=y}\right)_{y \in \mathbb{R}}$ defined by

$$
\mathbb{P}_{X_{t}=y}= \begin{cases}\mathbb{P}_{q-} & \text { if } \mu_{t}(y)=0, \\ \frac{1}{\mu(y)} \int_{q^{-}}^{q^{+}} \mathbb{P}_{q} \mathrm{~d} q & \text { otherwise }\end{cases}
$$

is a disintegration of $\mathbb{P}$ according to $X_{t}$.

For $\mu(y)=0$ the measure of both $A$ and $B$ for $\mathbb{P}_{X_{t}=y}$ is zero or one so that $A$ and $B$ are independent. In the other case, let us prove that at least one of the two events has measure zero or one. In fact, the quantiles of $\left.] q^{-}, q^{+}\right]$are mapped on $y$ by $G_{t}$. Hence, according to the criterion for every $i \leq k$ one of the two maps $G_{s_{i}}$ or $G_{u}$ is constant on ]$\left.q^{-}, q^{+}\right]$. Thus $G_{u}$ is constant or $\left(G_{s_{1}}, \ldots, G_{s_{k}}\right)$ is constant. Therefore $\mathbb{P}_{q}(A)$ or $\mathbb{P}_{q}(B)$ is constantly zero or one on $\left.] q^{-}, q^{+}\right]$. We have proved that $A$ and $B$ are independent with respect to $P_{X_{t}=y}$. This completes the proof of the first implication.

For the second implication suppose that the criterion is not satisfied so that there exist $s \leq t \leq u$ and $\left.q<q^{\prime} \in\right] 0,1\left[\right.$ with $G_{t}\left(q^{\prime}\right)=G_{t}(q):=y, G_{s}\left(q^{\prime}\right)>G_{s}\left(q^{\prime}\right):=x$ and $G_{u}\left(q^{\prime}\right)>G_{u}(q):=z$. In this case $\mathbb{P}\left(X_{t}=y\right)>0$. Let $q_{s}=F_{s}(x)$ and $q_{u}=F_{u}(z)$. Let also $q^{+}=F_{t}(y)$ and $q^{-}=\lim _{\varepsilon \rightarrow 0^{+}} F_{t}(y-\varepsilon)$ so that 
Peacocks Parametrised by a Partially Ordered Set

$$
q^{-}<\min \left(q_{s}, q_{u}\right) \leq \max \left(q_{s}, q_{u}\right)<q^{\prime} \leq q^{+} .
$$

We have on the one hand

$$
\mathbb{P}_{X_{t}=y}\left(X_{s} \leq x, X_{u} \leq z\right)=\frac{\min \left(q_{s}, q_{u}\right)-q^{-}}{q^{+}-q^{-}}
$$

and on the other hand

$$
\mathbb{P}_{X_{t}=y}\left(X_{s} \leq x, X_{u} \leq z\right)=\frac{q_{s}-q^{-}}{q^{+}-q^{-}} \quad \text { and } \quad \mathbb{P}_{X_{t}=y}\left(X_{s} \leq x, X_{u} \leq z\right)=\frac{q_{u}-q^{-}}{q^{+}-q^{-}} .
$$

Hence $\left\{X_{s} \leq x\right\}$ and $\left\{X_{u} \leq z\right\}$ are not conditionally independent given $\left\{X_{t}=y\right\}$. This finishes the proof of the second implication.

\subsubsection{Quantile martingales and characterisation of the Markov property}

Theorem 4 and 5 are the counterpart in the martingale setting of Proposition 2 and Proposition 3.

Theorem 4. Let $\left(\mu_{t}\right)_{t \in T}$ be a peacock indexed by a partially ordered set $(T, \leq)$. Assume moreover that the measures have expectation zero and $t \mapsto \mu_{t}$ is nondecreasing for the diatomic convex order. Then there exists $\left(X_{t}\right)_{t \in T}$ a martingale associated to $\left(\mu_{t}\right)_{t \in T}$.

Proof. According to paragraph 4.1.1, the elements of the canonical decomposition of the measures are in convex order. Hence we can replace the peacock by a onedimensional family of peacocks $\left(\theta_{-f^{t}(q), g^{t}(q)}\right)_{t \in T}$. Each of them can be associated with a martingale $\left(X_{t}^{q}\right)$ defined on the Wiener space as in paragraph 4.2.2. We consider the process on the probability space $[0,1] \times C([0,+\infty[)$ obtained using the conditioning in $q \in[0,1]$. It is a martingale with the correct marginal for every $t \in T$. We used the fact that convex combinations of martingale laws are martingale laws.

We call quantile martingale the martingale introduced during the proof of Theorem 4. In what follows we write $I^{t}(q)$ for the interval $\left[f^{t}(q), g^{t}(q)\right]$.

Theorem 5. With the notation of Theorem 4 the quantile martingale is Markovian if and only if the following criterion is satisfied for every $s \leq t \leq u$ and $q<q^{\prime} \in[0,1]$.

1. If $I^{t}(q)=I^{t}\left(q^{\prime}\right)$ it holds $I^{s}(q)=I^{s}\left(q^{\prime}\right)$ or $I^{u}(q)=I^{u}\left(q^{\prime}\right)$,

2. if $\left\{f^{t}(q)=f^{t}\left(q^{\prime}\right)\right.$ and $\left.g^{t}(q) \neq g^{t}\left(q^{\prime}\right)\right\}$ it holds

$I^{s}\left(q^{\prime}\right)=[0,0]$ or $\left\{f^{s}(q)=f^{s}\left(q^{\prime}\right)\right.$ and $g^{s}(q)=g^{t}(q)$ and $\left.g^{s}\left(q^{\prime}\right)=g^{t}\left(q^{\prime}\right)\right\}$ or $f^{t}\left(q^{\prime}\right)=f^{u}\left(q^{\prime}\right)$ or $I^{u}(q)=I^{u}\left(q^{\prime}\right)$,

3. if $\left\{f^{t}(q) \neq f^{t}\left(q^{\prime}\right)\right.$ and $\left.g^{t}(q)=g^{t}\left(q^{\prime}\right)\right\}$ it holds $I^{s}\left(q^{\prime}\right)=[0,0]$ or $\left\{g^{s}(q)=g^{s}\left(q^{\prime}\right)\right.$ and $f^{s}(q)=$ $f^{t}(q)$ and $\left.f^{s}\left(q^{\prime}\right)=f^{t}\left(q^{\prime}\right)\right\}$ or $g^{t}\left(q^{\prime}\right)=g^{u}\left(q^{\prime}\right)$ or $I^{u}(q)=I^{u}\left(q^{\prime}\right)$.

4. Nothing has to be satisfied in the case $\left\{f^{t}(q) \neq f^{t}\left(q^{\prime}\right)\right.$ and $\left.g^{t}(q) \neq g^{t}\left(q^{\prime}\right)\right\}$. 
Example 1 (Sufficient conditions). The criterion for the Markov property in Theorem 5 applies for instance in the following situations.

- The measures are continuous (without atom). This is settled in (4).

- The measures $\mu_{t}$ are continuous or $\delta_{0}$. If $\mu_{t}=\delta_{0}$ we check that the criterion is satisfied in (1) with $I^{s}(q)=I^{s}\left(q^{\prime}\right)=[0,0]$. The other case is (4).

- The measures are diatomic like in paragraph 4.2.2. For every $q<q^{\prime}$ and $t \in T$ it holds $I^{t}(q)=I^{t}\left(q^{\prime}\right)$ so that the criterion is satisfied in (1).

- The measures are $\mu_{t}=1 / 2\left(\theta_{-1,1+t}+\theta_{-1,2+t}\right)$ for $T=[0,1]$. The peacock satisfies the criterion in (2) where $f^{t}\left(q^{\prime}\right)=f^{u}\left(q^{\prime}\right)=-1$.

- The measures are $\mu_{t}=1 / 2\left(\theta_{-1-t, 1}+\theta_{-1-t, 2}\right)$ for $T=[0,1]$. For $q \leq 1 / 2<q^{\prime}$ the criterion is satisfied in (2) because it holds $\left\{f^{s}(q)=f^{s}\left(q^{\prime}\right)=-1-s\right.$ and $g^{s}(q)=$ $g^{t}(q)=1$ and $\left.g^{s}\left(q^{\prime}\right)=g^{t}\left(q^{\prime}\right)=2\right\}$.

Proof (Proof of Theorem 5). The proof is similar to the one of Proposition 3 even if more technical. In particular even if $\mu_{t}$ is continuous, the value of $X_{t}$ does not uniquely determine a trajectory. Nevertheless the law of the random trajectory is uniquely determined because it only depends on $q$ and $X_{t}$. In fact, the quantile $q$ is a function of $X_{t}$ so that as described in paragraph 4.2.2 the law of the future is contained in the present position $X_{t}$. In the general case when $\mu_{t}$ has atoms the process that is Markovian when conditioned on $q$ can loose the Markov property because $X_{t}$ does not uniquely determine $q$.

Suppose that $\left(X_{t}\right)_{t \in T}$ described above is not Markovian. We will show that the criterion is not satisfied. Let us consider a time $t \in T$ and $y \in \mathbb{R}$ so that $\left\{X_{t}=y\right\}$ denies the Markov property: the future is not independent from the past. Observe that the previous remarks on continuous measures show that $y$ must be an atom of $\mu_{t}$. Moreover $y=0$ is not possible because it would mean $X_{s}=0$ for $s \leq t$. Hence the past would be determined by the present so that no information on the past can change the law of the future.

Without loss of generality, we assume $X_{t}=y<0$. Let $Q=\left\{q \in[0,1], f^{t}(q)=y\right\}$ be the interval of quantiles mapped in $y$. On $Q$ the density of probability for the value of $q$ conditioned on $X_{t}=y$ is proportional to $\frac{g^{t}}{g^{t}-f^{t}}$. As we supposed that the Markov property does not hold there exists an integer $k$ and indices $s_{1}, \ldots, s_{k} \leq t$ such that $\left(X_{s_{1}}, \ldots, X_{s_{k}}\right)$ and $X_{u}$ are not conditionally independent given $\{q \in Q\} \cap\left\{X_{t}=y\right\}=$ $\left\{X_{t}=y\right\}$. As these random variables are independent with respect to the conditional probabilities $\mathbb{P}_{\{q\} \cap\left\{X_{t}=y\right\}}$ for any $q \in Q$, there exist two quantiles for which both the laws of the past and of the future are different respectively ${ }^{2}$. Let $q_{1}, q_{2} \in Q$ with $q_{1}<q_{2}$ be such quantiles.

Concerning the future first, the law $\beta_{i}$ of $X_{u}$ is the one of a Brownian motion starting in $y=f^{t}\left(q_{i}\right)<0$ and stopped when hitting $f^{u}\left(q_{i}\right) \leq y$ or $g^{u}\left(q_{i}\right)>0$. Different future laws $\beta_{i}$ are obtained for $i \in\{1,2\}$. Therefore $f^{u}\left(q_{2}\right)<y$ and $\left[f^{u}\left(q_{1}\right), g^{u}\left(q_{1}\right)\right] \neq\left[f^{u}\left(q_{2}\right), g^{u}\left(q_{2}\right)\right]$.

\footnotetext{
${ }^{2}$ It is a general fact that if $\mathfrak{p}$ and $\mathfrak{f}$, a past and a future map defined on $Q$ are both nonconstant, there exist $q_{1}, q_{2} \in Q$ such that $\mathfrak{p}\left(q_{1}\right) \neq \mathfrak{p}\left(q_{2}\right)$ and $\mathfrak{f}\left(q_{1}\right) \neq \mathfrak{f}\left(q_{2}\right)$.
} 
We consider now the past. For some $s \in\left\{s_{1}, \ldots, s_{k}\right\}$ we write $\alpha_{i}$ the law of $X_{s}$ given $\left\{q_{i}\right\} \cap\left\{X_{t}=y\right\}$. It is the law of a Brownian motion stopped when it hits $\left\{f^{s}\left(q_{i}\right), g^{s}\left(q_{i}\right)\right\}$ conditioned on the fact that it hits $y=f^{t}\left(q_{i}\right)$ before $g^{t}\left(q_{i}\right)$. Recall also $f^{t}\left(q_{i}\right) \leq f^{s}\left(q_{i}\right) \leq 0 \leq g^{s}\left(q_{i}\right) \leq g^{s}\left(q_{i}\right)$. The support of $\alpha_{i}$ has cardinal one or two. It is one if and only if $I^{s}\left(q_{i}\right)=[0,0]$ or $g^{s}\left(q_{i}\right)=g^{t}\left(q_{i}\right)$ and then $\alpha_{i}$ is the Dirac mass in 0 or $f^{s}\left(q_{i}\right)$ respectively. If the support of $\alpha_{i}$ has two elements these are $\left\{f^{s}\left(q_{i}\right), g^{s}\left(q_{i}\right)\right\}$ and $\alpha_{1}=\alpha_{2}$ if and only if $g^{t}\left(q_{1}\right)=g^{t}\left(q_{2}\right)$ and $I^{s}\left(q_{1}\right)=I^{s}\left(q_{2}\right)$. If $g^{t}\left(q_{1}\right) \neq g^{t}\left(q_{2}\right)$ the only possibility for $\alpha_{1}=\alpha_{2}$ is that the supports are reduced to one point. Note now that if $\alpha_{1}=\alpha_{2}$ the support of those measures uniquely determine $I^{S}\left(q_{1}\right)$ and $I^{S}\left(q_{2}\right)$ in both cases $g^{t}\left(q_{1}\right)=g^{t}\left(q_{2}\right)$ or $g^{t}\left(q_{1}\right) \neq g^{t}\left(q_{2}\right)$. But for $i \in\{1,2\}$ the law of $\left(X_{s_{1}}, \ldots, X_{s_{k}}\right)$ given $\left\{q_{i}\right\} \cap\left\{X_{t}=y\right\}$ is uniquely determined by $\left\{I^{s}\left(q_{i}\right)\right\}_{s \in\left\{s_{1} \ldots, s_{k}\right\}}$. As we supposed that these laws are different for $i=1$ or $i=2$, there exists $s \in\left\{s_{1}, \ldots, s_{k}\right\}$ such that $I^{s}\left(q_{2}\right) \neq[0,0]$ and $\left\{g^{s}\left(q_{1}\right) \neq g^{t}\left(q_{1}\right)\right.$ or $g^{s}\left(q_{2}\right) \neq$ $g^{t}\left(q_{2}\right)$ or $\left.f^{s}\left(q_{1}\right) \neq f^{t}\left(q_{1}\right)\right\}$ in the case $g^{t}\left(q_{1}\right) \neq g^{t}\left(q_{2}\right)$ and $I^{t}\left(q_{1}\right) \neq I^{t}\left(q_{2}\right)$ in the case $g^{t}\left(q_{1}\right)=g^{t}\left(q_{2}\right)$.

In summary, let $y<0$ be an atom of $\operatorname{Law}\left(X_{t}\right)$ such that the condition $\left\{X_{t}=y\right\}$ denies the Markov property of the quantile martingale. Concerning the future we have proved $f^{u}\left(q_{2}\right)<y$ and $\left[f^{u}\left(q_{1}\right), g^{u}\left(q_{1}\right)\right] \neq\left[f^{u}\left(q_{2}\right), g^{u}\left(q_{2}\right)\right]$. Concerning the past, we have proved $I^{s}\left(q_{2}\right) \neq[0,0]$ and $\left\{g^{s}\left(q_{1}\right) \neq g^{t}\left(q_{1}\right)\right.$ or $g^{s}\left(q_{2}\right) \neq g^{t}\left(q_{2}\right)$ or $f^{s}\left(q_{1}\right) \neq$ $\left.f^{t}\left(q_{1}\right)\right\}$ in the case $g^{t}\left(q_{1}\right) \neq g^{t}\left(q_{2}\right)$ and $I^{t}\left(q_{1}\right) \neq I^{t}\left(q_{2}\right)$ in the case $g^{t}\left(q_{1}\right)=g^{t}\left(q_{2}\right)$. Symmetric conclusions happen in the symmetric situation $y>0$. Hence one can carefully check that at least (1), (2) or (3) is not correct for the choice $\left(q, q^{\prime}\right)=$ $\left(q_{1}, q_{2}\right)$. In fact if $g^{t}\left(q_{1}\right)=g^{t}\left(q_{2}\right)$ the criterion is not satisfied in (1). If $g^{t}\left(q_{1}\right)=$ $g^{t}\left(q_{2}\right)$, it is not satisfied in (2). Finally we have proved that if the peacock satisfies the criterion the quantile martingale is Markovian.

Conversely, we assume that the criterion is not satisfied and will prove that the process is not Markovian. It is enough to assume that the criterion is not satisfied in (1) or (2). For $s \leq t \leq u$ and $q \in Q=\left(f^{t}\right)^{-1}\{y\}$ we denote as before $\alpha(q)$ the law of $X_{s}$ given $\{q\} \cap\left\{X_{t}=y\right\}$ and $\beta(q)$ the law of $X_{u}$ given the same condition. Let $q, q^{\prime} \in Q$ such that the criterion is not satisfied. If the criterion is not satisfied in (1) we have $I^{u}(q) \neq I^{u}\left(q^{\prime}\right)$ and we can assume $f^{u}\left(q^{\prime}\right)<f^{u}(q) \leq y$ (if not $g^{u}\left(q^{\prime}\right)>$ $g^{u}(q) \geq g^{t}(q)=y^{\prime}$ and we can consider $y^{\prime}$ in place of $\left.y\right)$. Therefore $\beta(q) \neq \beta\left(q^{\prime}\right)$ and $\alpha(q) \neq \alpha\left(q^{\prime}\right)$ in the two cases. The joint law of $\left(X_{s}, X_{u}\right)$ given $\left\{X_{t}=y\right\}$ is

$$
\pi:=Z^{-1} \int_{Q} \alpha(q) \times \beta(q) \frac{g^{t}(q)}{g^{t}(q)-f^{t}(q)} \mathrm{d} q
$$

where $Z=\int_{Q} \frac{g^{t}(q)}{g^{t}(q)-f^{t}(q)} \mathrm{d} q$. We will prove that it is not the product of two probability measures, which will be enough for the implication. Recall that the support of $\alpha(q)$ and $\beta(q)$ are included in $\left\{f^{s}(q), g^{s}(q)\right\}$ and $\left\{f^{u}(q), g^{u}(q)\right\}$ respectively. The functions $f$ are nonincreasing and left-continuous. The functions $g$ are nondecreasing and left-continuous. In case (1) the measures $\alpha(q) \neq \alpha\left(q^{\prime}\right)$ and $\beta(q) \neq \beta\left(q^{\prime}\right)$ are not only different but their supports are also different. Hence one is easily convinced with a picture in $\mathbb{R}^{2}$ that $\pi$ has not the support of a product measure. This argument does not work if (2) is denied because $\alpha(q)$ and $\alpha\left(q^{\prime}\right)$ may have the 
same support and be different. In fact they are different if and only if the support is made of the two points $f^{s}(q)=f^{s}\left(q^{\prime}\right)$ and $g^{s}(q)=g^{s}\left(q^{\prime}\right)$. With a simple Bayes formula the mass of $f^{s}(q)$ with respect to $\alpha(q)$ can be computed to be $\left(g^{s}(q) / g^{s}(q)-f^{s}(q)\right)\left(g^{t}(q)-f^{t}(q) / g^{t}(q)\right)$ and the same formula with primed letters holds for $\alpha\left(q^{\prime}\right)$. Note that the four quantities are the same except $g^{t}\left(q^{\prime}\right)>g^{t}(q)$. As $\beta(q) \neq \beta\left(q^{\prime}\right)$ and recalling the left continuity of $f^{u}$ and $g^{u}$ it follows that the conditional law of $X_{u}$ with respect to $\left\{X_{t}=y\right.$ and $\left.X_{s}=f^{s}(q)\right\}$ is different to the conditional law of $X_{u}$ with respect to $\left\{X_{t}=y\right.$ and $\left.X_{s}=g^{s}(q)\right\}$. Finally $\pi$ is not a product measure and the martingale in not Markovian.

\subsection{Questions}

Even though Problem 7b is solved in [8], it is still an open question whether the full Kellerer theorem for measures on $\mathbb{R}^{d}$ hold, where "full" means with the Markov property. The following questions are rather related to our approach of Problem 7a in Section 4.3. To solve them may however bring some useful new ideas to Problem $7 \mathrm{~b}$.

- Let $t \mapsto \mu_{t}$ be nondecreasing for the stochastic order. Does it exist an associated process $\left(X_{t}\right)_{t \in T}$ that is Markovian? Recall that Proposition 3 is an exact account on the question whether the quantile process associated to $\left(\mu_{t}\right)_{t}$ is Markovian.

- Let $\left(\mu_{t}\right)_{t \in[0,1]}$ be a family of real measures. For any sequence of partitions of $[0,1]$ we describe a procedure. We associate to the partition $0=t_{0} \leq \cdots \leq t_{N}=1$ the Markovian process $\left(X_{t}\right)_{t \in[0,1]}$ constant on any $\left[t_{k}, t_{k+1}\left[\right.\right.$ such that $\operatorname{Law}\left(X_{t}\right)=$ $\operatorname{Law}\left(X_{t_{k}}\right)$, and $\operatorname{Law}\left(X_{t_{k}}, X_{t_{k+1}}\right)$ is a quantile coupling. Under ad hoc general conditions on the peacock and the type of convergence, does it exist a sequence of partitions such that the sequence of processes converge to a Markovian limit process with marginal $\mu_{t}$ at any $t \in[0,1]$ ? Is the Markovian limit unique? Is for instance the continuity of the peacock sufficient for these properties? This makes precise a question at the end of [11]. See this paper and also [6] for the same approach in the case of martingales.

- If $t \mapsto \mu_{t}$ is nondecreasing for the diatomic convex order $\preceq_{D C}$, does it exist an associated Markovian martingale? We proved in Theorem 5 that such a martingale can not systematically be the quantile martingale.

Of course the first and the third question have likely the same answer, yes or no. In the case $T=[0,1]$ the second question suggests an approach for the first question. Recall that it is wrong that limit of Markovian processes are Markovian.

\section{References}

1. M. Beiglböck, M. Huesmann, and F. Stebegg. Root to Kellerer. preprint, 2015. 
2. M. Beiglböck and N. Juillet. On a problem of optimal transport under marginal martingale constraints. to appear in Ann. Probab., Aug. 2012.

3. R. Cairoli and J. B. Walsh. Stochastic integrals in the plane. Acta Math., 134:111-183, 1975.

4. R. G. Douglas. On extremal measures and subspace density. Michigan Math. J., 11:243-246, 1964.

5. J. A. Fill and M. Machida. Stochastic monotonicity and realizable monotonicity. Ann. Probab., 29(2):938-978, 2001.

6. P. Henry-Labordere, X. Tan, and N. Touzi. An Explicit Martingale Version of the Onedimensional Brenier's Theorem with Full Marginals Constraint. preprint, Feb. 2014.

7. F. Hirsch, C. Profeta, B. Roynette, and M. Yor. Peacocks and associated martingales, with explicit constructions, volume 3 of Bocconi \& Springer Series. Springer, Milan, 2011.

8. F. Hirsch and B. Roynette. On $\mathbb{R}^{d}$-valued peacocks. ESAIM Probab. Stat., 17:444-454, 2013.

9. F. Hirsch, B. Roynette, and M. Yor. Kellerer's theorem revisited. In Springer, editor, Asymptotic Laws and Methods in Stochastics. Volume in Honour of Miklos Csorgo, Fields Institute Communications Series, 2014.

10. F. Hirsch and M. Yor. Looking for martingales associated to a self-decomposable law. Electron. J. Probab., 15:no. 29, 932-961, 2010.

11. N. Juillet. Stability of the shadow projection and the left-curtain coupling. to appear in Ann. Inst. Henri Poincaré Probab. Stat., 2014.

12. T. Kamae and U. Krengel. Stochastic partial ordering. Ann. Probab., 6(6):1044-1049 (1979), 1978.

13. T. Kamae, U. Krengel, and G. O'Brien. Stochastic inequalities on partially ordered spaces. Ann. Probab., 5:899-912, 1977.

14. H. G. Kellerer. Markov-Komposition und eine Anwendung auf Martingale. Math. Ann., 198:99-122, 1972.

15. B. Pass. On a class of optimal transportation problems with infinitely many marginals. SIAM J. Math. Anal., 45(4):2557-2575, 2013.

16. V. Strassen. The existence of probability measures with given marginals. Ann. Math. Statist., 36:423-439, 1965.

17. E. Wong and M. Zakai. Martingales and stochastic integrals for processes with a multi- dimensional parameter. Z. Wahrscheinlichkeitstheor. Verw. Geb., 29:109-122, 1974. 